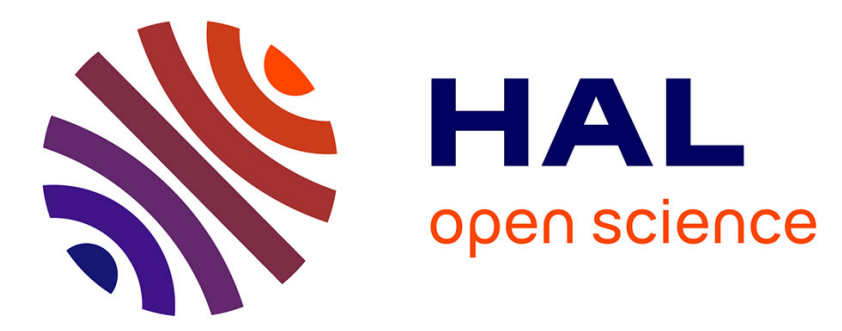

\title{
Rotational Doppler shift from a rotating rod
}

Olivier Emile, Janine Emile, Christian Brousseau, Tangi Le Guennic, Pu Jian, Guillaume Labroille

\section{To cite this version:}

Olivier Emile, Janine Emile, Christian Brousseau, Tangi Le Guennic, Pu Jian, et al.. Rotational Doppler shift from a rotating rod. Optics Letters, 2021, 46 (15), pp.3765-3768. 10.1364/OL.435058 . hal-03330279

\section{HAL Id: hal-03330279 \\ https://hal.science/hal-03330279}

Submitted on 20 Sep 2021

HAL is a multi-disciplinary open access archive for the deposit and dissemination of scientific research documents, whether they are published or not. The documents may come from teaching and research institutions in France or abroad, or from public or private research centers.
L'archive ouverte pluridisciplinaire HAL, est destinée au dépôt et à la diffusion de documents scientifiques de niveau recherche, publiés ou non, émanant des établissements d'enseignement et de recherche français ou étrangers, des laboratoires publics ou privés. 


\title{
Rotational Doppler shift from a rotating rod
}

\author{
Olivier Emile ${ }^{1,}$, Janine Emile $^{2}$, Christian Brousseau $^{3}$, Tangi le Guennic ${ }^{4}$, Pu Jian ${ }^{4}$, And \\ GUILLAUME LABROILLE ${ }^{4}$
}

${ }^{1}$ Université de Rennes 1, Campus de Beaulieu, F-35000 Rennes, France

${ }^{2}$ Université de Rennes 1, CNRS IPR UMR 6251, F-35000 Rennes, France

${ }^{3}$ Université de Rennes 1, CNRS IETR UMR 6164, F-35000 Rennes, France

${ }^{3}$ CAILabs, 38 boulevard Albert $1^{\text {er }}$, F-35200 Rennes, France

*olivier.emile@univ-rennes1.fr

Compiled July 2, 2021

This letter reports the observation of a rotational Doppler effect from a rotating rod illuminated by a fundamental Gaussian laser beam. More specifically, we decompose the transmitted light behind the rotating rod into Laguerre-Gaussian modes and investigate the associated frequency shifts. The main contributing modes correspond to modes having the same rotational symmetry as the rotating object. Besides, their shifts equal the topological charge of the beam times the rotational frequency of the object. Potential applications in pattern recognition and rotation identification are then considered. (๑) 2021 Optical Society of America

http://dx.doi.org/10.1364/ao.XX.XXXXXX

The Doppler frequency shift occurs when a wave source and a receiver are moving regarded to each other [1]. It has many applications ranging from astrophysics [2], Doppler velocimetry [3], laser cooling [4] to radar systems [5]. Conversely, in the case of rotations, there is also a "so called" rotational Doppler effect related to the angular momentum (orbital or spin) of the wave [6]. Whereas the linear Doppler effect requires a change of the wave vector, the rotational Doppler effect is linked to a variation of the angular momentum [7]. This limits, up to now, rotational observations to waves carrying Spin Angular Momentum (SAM) or Orbital Angular Momentum (OAM) to specific situations. In particular, it has been reported for waves transmitted through specific elements [8], or scattered [9, 10] or reflected on suitable surfaces such as phase conjugated mirrors [11], scattered from ion-acoustic vortices in laser plasma [12], metasurfaces and helicoidal reflectors [13, 14] or prisms [15]. It thus also greatly restricts the potential applications. One may then wonder whether such rotational Doppler effect could be evidenced using ordinary light and a simple rotating object. The aim of this letter is thus to investigate the transmitted light behind a rotating metallic rod that could mimic a two-blade propeller and to consider the possible frequency variation of the transmitted light.

Usually, target recognition experiments using OAM beams examine the interaction of light and object, that have the same rotational symmetry [16]. Here, conversely, a simple rod is illuminated by a fundamental Gaussian beam, as sketched in
Fig. 1. The eye safe $(\lambda=1550 \mathrm{~nm})$ free space propagation of the fundamental mode from a CW fiber laser (CEFL, Keopsys, connected to a single mode fiber SMF-28) is mode-matched to the fundamental mode of a commercially available space division multiplexing unit based on Multi-Plane Light Conversion (MPLC) [17], a patented technology developed by Cailabs in 2013. The MPLC technology allows to perform any unitary spatial transform. Theoretically, any unitary spatial transform can be implemented by a succession of transverse phase profiles separated by free space propagation for optical Fourier transforms. The MPLC cavity is formed by one mirror and one reflective phase plate, implementing the successive phase profiles and optical transforms, to realize any unitary spatial transform, with low optical losses and high modal selectivity. The MPLC has been here configured to multiplex/demultiplex Hermite-Gaussian modes (HG). However, in another configuration, a MPLC can address any spatial mode profile, especially Laguerre-Gaussian (LG) beams with high fidelity.

Since this unit multiplexes or demultiplexes into HG modes [18] that have cartesian symmetry we use a mode converter based on cylindrical lenses [19] to decompose the transmitted light into LG modes that have rotational symmetry. The MPLC demultiplexing unit and the mode converter are here used to decompose the transmitted light on an LG basis. These modes are noted $\mathrm{LG}_{n}^{\ell}$ hereafter, where $n$ is the radial order, and $\ell$ is the azimuthal order or topological charge. Let us recall that a LG mode has an azimuthal dependence that writes $\exp (i \ell \theta), \theta$ being the azimuthal angle [6].

In order to check that the outputs of the spatial multiplexer corresponds to LG beams, we use the principle of reversibility of light, and plug directly the output of the laser into the in/output of the various modes of the spatial multiplexer. We then use the double slit experiment for OAM beams [20], to characterize the free space propagation of the output of the spatial multiplexer. Actually the twisting order of the interference fringes of an OAM beam compared with the fundamental beam equals the topological charge of the beam. We could verify that these modes actually correspond to LG modes.

A $1 \mathrm{~mm}$-diameter copper rod is rotated perpendicular to its axis (see Fig. 1) at an adjustable frequency $v_{\text {rot }}$ thanks to a homemade hollow shaft supporting the rod and driven by a belt and a motor. The middle of the rod corresponds to the 


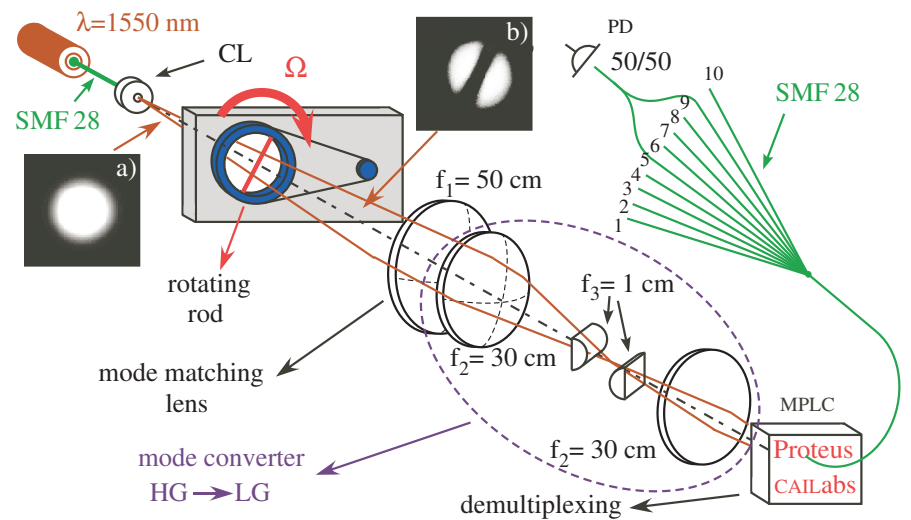

Fig. 1. Experimental set-up. CW laser source: $\lambda=1550$ $\mathrm{nm}$. CL: Collimating lens at the output of the fiber. $f_{1}=50 \mathrm{~cm}$ : mode matching lens. $f_{2}=30 \mathrm{~cm}$ : focusing lenses; distance between them $60 \mathrm{~cm} . \mathrm{f}_{3}=1 \mathrm{~cm}$ : cylindrical lenses acting as a $\pi / 2$ converter; distance between them $1.41 \mathrm{~cm}$. Proteus: space division multiplexer generating Hermite-Gaussian modes. PD: photodiode. 50/50: coupler with a 50/50 intensity repartition. Inset: Photograph of the beam profile a) before the rod, b) after the rod.

rotation axis of the shaft and also to the optical axis of the whole experiment. The intensity of each LG mode is monitored on a photodiode. An example of the decomposition of the light transmitted through the experimental set up is displayed in Fig. 2, without (Fig. 2a) and with (Fig. 2b) the rod. In Fig. 2b, the rod is not rotating and is set vertically.
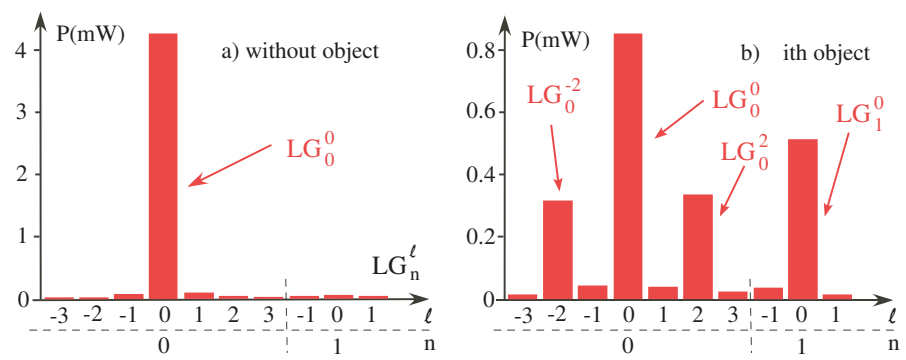

Fig. 2. Intensity decomposition of the free space laser propagation in LG modes, a) without, b) with the non-rotating rod. The first seven modes correspond to a radial number $n=0$, the next three modes correspond to $n=1$.

One can first note that without the rod the mode matching condition between the fiber laser output and the spatial multiplexer is properly fulfilled (see Fig.2a). There is nearly $95 \%$ of the incoming light that is injected in the fundamental mode $\left(\mathrm{LG}_{0}^{0}\right)$ of the spatial multiplexer. As the rod is inserted, the intensity in the fundamental mode decreases, and the intensity of the other modes increases (see Fig.2b). At first sight, it could be strange that the contributions of the $\mathrm{LG}_{0}^{-1}$ and the $\mathrm{LG}_{0}^{1}$ modes to the decomposition are very low. Indeed, the transmitted light shape intensity shown in Fig. 1 is similar to a $\mathrm{HG}_{1}^{0}$ that is a linear combination of the $\mathrm{LG}_{0}^{-1}$ and the $\mathrm{LG}_{0}^{1}$. One could expect these modes to dominate. However, in a $\mathrm{HG}_{1}^{0}$ mode, the two intensity lobes are out of phase [18] whereas in our experiment, the intensity lobes of the transmitted light have the same phase. They thus play no role in the decomposition.
This can be demonstrated more rigorously using symmetry arguments. The occulting rod has a $\pi$ rotation symmetry, whereas the $\mathrm{LG}_{0}^{-1}$ and the $\mathrm{LG}_{0}^{1}$ modes have a $2 \pi$ rotation symmetry only. They thus logically do not contribute into the decomposition of the transmitted light. Besides, the same argument also holds for $\mathrm{LG}_{0}^{-\ell}$ and the $\mathrm{LG}_{0}^{\ell}$ modes for odd values of $\ell$. On the contrary, $\mathrm{LG}_{0}^{-2}$ and the $\mathrm{LG}_{0}^{2}$ modes have the same $\pi$ rotational symmetry as the rod. They thus mainly contribute to the decomposition of the transmitted light. This is in very good agreement with the experimental observations of Fig. $2 b$. This clearly demonstrates that a decomposition in LG modes enables to identify the rotational symmetry of the object. It is worth noting that such a modal decomposition has also been used to identify the opening angle of objects [21].

This first part of the experiment was dedicated to the identification of the symmetries which is a prerequisite when the symmetries are unknown. Once we have identified them, we concentrate on the dominant modes of the decomposition only. Among them, the $\mathrm{LG}_{0}^{0}$ mode has no azimuthal dependence whereas $\mathrm{LG}_{0}^{-2}$ and $\mathrm{LG}_{0}^{2}$ vary as $\exp (-i 2 \theta)$ and $\exp (i 2 \theta)$ respectively. As the rod is rotated by an angle $\theta_{0}$, the pattern of the transmitted light is also rotated by an angle $\theta_{0}$. This is equivalent to a change of the azimuthal angle's origin. It then leaves the phase of the $\mathrm{LG}_{0}^{0}$ mode unchanged, whereas the azimuthal dependence of the phase of the $\mathrm{LG}_{0}^{-2}$ and $\mathrm{LG}_{0}^{2}$ modes now writes

$$
\exp \left(-i 2\left(\theta-\theta_{0}\right)\right) \text { and } \exp \left(i 2\left(\theta-\theta_{0}\right)\right)
$$

respectively. As the rod is rotated at a constant frequency $v_{r o t}$, the angle $\theta_{0}$ varies with time $t$ and equals $\theta_{0}=2 \pi v_{\text {rot }} t$. According to equation 1 , this is equivalent to a frequency shift $\Delta v_{\ell}$ of the light frequency of the $\mathrm{LG}_{0}^{\ell}$

$$
\Delta v_{\ell}=\ell v_{r o t}
$$

with $\ell= \pm 2$ here. This is nothing but the rotational Doppler effect formula [6]. Noteworthy, in stimulated Brillouin scattering the change of OAM is $2 \hbar \ell$ per photon. In this case, rotational Doppler shift is doubled due to backscattering on rotating acoustical helices [22]. The frequency shift is equal to the product of the rotation frequency of the object times the change of OAM of light. The frequency of the mode having the same direction of rotation of the phase as the direction of rotation of the object is decreased while the frequency of the other mode is increased.

Fig. 3 shows the de-multiplexed intensities of the $\mathrm{LG}_{0}^{-2}, \mathrm{LG}_{0}^{2}$ and $L_{0}^{0}$ modes recorded on the photodiodes, while the rod is rotating at a constant frequency $v_{r o t}=2 \mathrm{~Hz}$. It is worth noting that, on Fig. 3b, c and d, the intensities of these three modes are nearly constant. The residual modulation probably corresponds to a slight misalignment of the axis of the shaft and of the rod, in relation to the optical axis. Let us now mix the different de-multiplexed modes together or with a reference signal originating from the laser source. The mixing of light with different topological charges has been widely used in rotational Doppler measurement [23]. However, here, each mode with a given topological charge is isolated. This enables the mixing with a reference or the mixing with other modes in a very versatile way. It can thus discriminate between intensity modulations and beat frequencies. Moreover, this mixing can be easily performed since the light output of each multiplexed channel is coupled into fibers, taking advantage of commercially available fiber attenuators and couplers at telecommunication wavelengths. 

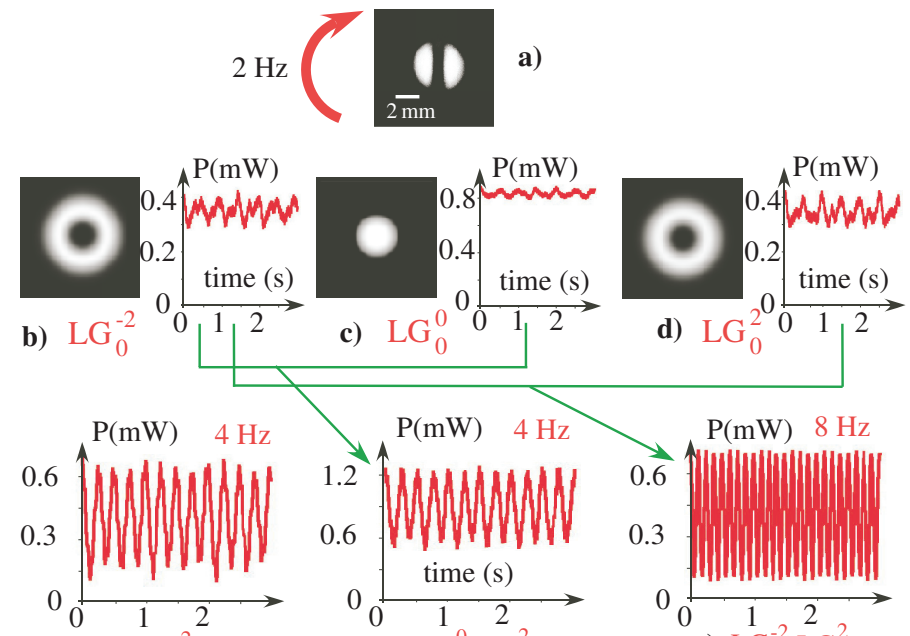

e) $\mathrm{ref}+\mathrm{LG}_{0}^{2}$

f) $\mathrm{LG}_{0}^{0}+\mathrm{LG}_{0}^{2}$

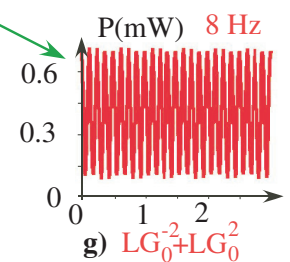

Fig. 3. a) Picture of the transmitted light. Picture and intensity at the output of the MPLC of the b) $\mathrm{LG}_{0}^{-2}$, c) $\mathrm{LG}_{0}^{2}$ and d) $\mathrm{LG}_{0}^{0}$. Beat frequency between e) the laser source and the output corresponding to the $\mathrm{LG}_{0}^{2}$ mode, $\mathrm{f}$ ) the output corresponding to the $\mathrm{LG}_{0}^{2}$ and $\mathrm{LG}_{0}^{0}$ modes, $\mathrm{g}$ ) the output corresponding to the $\mathrm{LG}_{0}^{-2}$ and $\mathrm{LG}_{0}^{2}$ modes.

Fig. 3e shows the intensity of the coupling between a reference signal from the laser source and the output of the spatial multiplexer corresponding to the de-multiplexed $\mathrm{LG}_{0}^{2}$ mode. There is clearly a strong modulation corresponding to a beat frequency $v_{\text {beat }}$ between the two signals. It is equal to twice the rotation frequency of the rod $v_{\text {rot }}$. The same conclusion also holds for the beat frequency between the $\mathrm{LG}_{0}^{0}$ and the $\mathrm{LG}_{0}^{2}$ modes (see Fig. 3f). It is also true for the coupling between the reference and the $\mathrm{LG}_{0}^{-2}$ mode, and for the coupling between the $\mathrm{LG}_{0}^{-2}$ and the $\mathrm{LG}_{0}^{0}$ modes. This modulation couldn't be attributed to a modulation of the intensity of the mode, as it would be the case, for example, for a point like detector, since the intensity of each mode is constant (see Fig. 3b, c, d). It clearly evidences that the frequencies of the $\mathrm{LG}_{0}^{-2}$ and $\mathrm{LG}_{0}^{2}$ modes are shifted by a $4 \mathrm{~Hz}$ frequency regarded to the incident beam. Besides, the signal corresponding to the coupling of the $\mathrm{LG}_{0}^{-2}$ and $\mathrm{LG}_{0}^{2}$ modes is modulated at a frequency of $8 \mathrm{~Hz}$ (see Fig. $3 \mathrm{~g}$ ), corresponding to a beat frequency of $8 \mathrm{~Hz}$. One mode is shifted by $4 \mathrm{~Hz}$, whereas the other is shifted by a $-4 \mathrm{~Hz}$.

As the rotation frequency of the rod $v_{r o t}$ is varied, the beat frequencies $v_{\text {beat }}$ must change accordingly. Fig. 4 displays the measured beat frequencies $v_{\text {beat }}$ versus $v_{\text {rot }}$. It is worth noting that the beat frequency between (i) the reference and the $\mathrm{LG}_{0}^{2}$ or the $\mathrm{LG}_{0}^{-2}$ modes, and (ii) the $\mathrm{LG}_{0}^{0}$ and the $\mathrm{LG}_{0}^{2}$ or the $\mathrm{LG}_{0}^{-2}$ varies linearly with $v_{\text {rot }}$. The slope $\alpha$ equals 2 . The beat frequency between the $\mathrm{LG}_{0}^{-2}$ and $\mathrm{LG}_{0}^{2}$ modes is also linear with a slope $\alpha$ equal to 4 . Obviously and unambiguously, the frequency of the two $\mathrm{LG}_{0}^{\ell}$ modes, with $\ell= \pm 2$ are shifted by $v=2 v_{\text {rot }}$ which is in agreement with Eq. 2 and with the expected rotational Doppler shift. One mode is shifted by $2 v_{\text {rot }}$, and the other mode is shifted by $-2 v_{r o t}$. The main uncertainty of our measurements relies on the determination of $v_{\text {rot }}$ which has been calibrated versus the supplied current. It is estimated to $0.1 \mathrm{~Hz}$. It induces a uncertainty of $0.2 \mathrm{~Hz}$ on the beat frequency with the reference beam and a uncertainty of $0.4 \mathrm{~Hz}$ on the beat frequency between the two LG modes (see Fig. 4). It is higher than the $0.02 \mathrm{~Hz}$ uncertainty linked to the acquisition time (50 s).

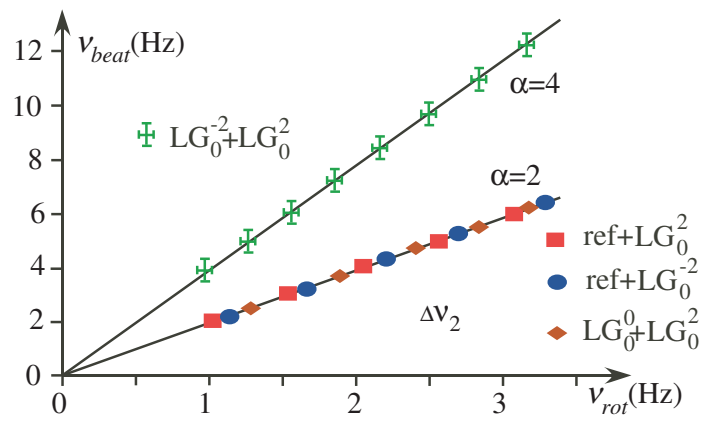

Fig. 4. Variation of the beat frequency $\Delta v_{\text {beat }}$ between the modes versus the rotation frequency $v_{r o t}$ of the rod. $\alpha$ is the is the proportionality factor between the beat frequency and the rotation frequency. The size of the points corresponds to the error bars.

The rotational Doppler effect has been evidenced here on the transmission of a fundamental Gaussian beam, in the most general configuration. There is no need to know the rotational symmetry of the object beforehand. It could have also been observed in reflection. For example, let us imagine to replace the rod by a dielectric reflecting object with the same dimensions. The transmission after such a mirror would be the same as the transmission after the rod in our experimental configuration. The reflected light on the mirror, neglecting diffraction, would be the complementary of the transmitted light regarded to the fundamental beam. Then the $|\ell|=2$ modes would have nonzero contribution to the reflected signal as well. As the mirror is set into rotation, the $|\ell|=2$ reflected modes would experience a rotational Doppler shift, just like the transmitted ones. This rotational Doppler shift in rotation could also be easily detected.

The observation of a rotational Doppler shift in our configuration is not limited to a $\pi$ rotational symmetry, but it can be generalized to any object having a $2 \pi / \ell$ rotational symmetry and to backscattering from rotating helical entities having $(2 \pi) /(2 \ell)$ rotational symmetry alike proteins and spiral index gratings [24]. It can be performed either in transmission or in reflection. The main dominant LG modes in the de-multiplexed intensity signals should then correspond to the rotational symmetry of the object and carry a topological charge equal to $\pm \ell$. On the one hand, it will enable clear identification of the rotational symmetry of the object. On the other hand, the associated rotational Doppler shift would be equal to the rotational frequency times the topological charge of the mode (doubled topological charge for backscattering) and will provide information on its dynamical behaviour. As the linear Doppler shift leads to a change of the linear velocity, a single rotational Doppler shift also leads to a change of the rotational velocity. The shift can thus be measured in a dual way, i.e. on the rotating velocity itself. Indeed, as pointed out by Padgett [25], the rotational Doppler effect is linked to the power of the associated torque. It results in a change of the frequency and thus in a change of the total energy of the field. Due to energy conservation [26], this change of energy of light must be compensated for a change of kinetic energy of the object. Unfortunately in our case, the frequency of the $\mathrm{LG}_{0}^{2}$ mode experiences a $2 v_{\text {rot }}$ that is annihilated by a shift of the frequency of the $\mathrm{LG}_{0}^{-2}$ mode by $-2 v_{\text {rot }}$. There is thus no change of energy of the rotating object. 
This kind of experiment, dedicated to the observation of the rotational Doppler shift, is not limited to optics but could be generalized to any electromagnetic wave carrying OAM, ranging from radio waves [27] to X-ray waves [28], and to any wave in general such as electrons [29], neutrons [30] or acoustic waves [31]. Among the various applications, special care should be given to the radio waves. Radar imaging and object identification in transmission (bistatic configuration) or in reflexion (monostatic radar) could be enriched with rotational frequency information such as the one obtained by the method described in this letter. This could be especially important for example in the identification, classification and rotation measurements, either in propellers or reactors in aeronautics, or in ships and submarines in sea transport.

To conclude, we have evidenced a rotational Doppler frequency shift from a fundamental mode transmitted behind a partially occulting rotating rod. The transmitted beam has been decomposed on a Laguerre-Gaussian basis. The predominant modes in the decomposition bear the same rotational symmetry as the rotating object, enabling its identification. The beat frequencies between the modes give unambiguously access the rotation frequency of the object, via the frequency shifts of the Laguerre-Gaussian modes. The method is valid for any object having a rotational symmetry which does not need to be known a priori.

This kind of rotation identification that could be generalized to any rotating system could also find applications in biology and medicine. Actually, there is an increasing need to find methods to pump or mix tiny amounts of analytes in biofluid media and microfluidics [32], via micro motors. A MPLC-based spatial multiplexer could be coupled to the output of a microscope to demultiplex the transmitted or reflected light in OAM modes, in order to identify symmetries and fully characterize the micro motor rotation. Such rotation identification could be generalized to the nano-world, in particular towards proteins and viruses recognition, and their rotational power [33], using OAM and electron microscopes.

\section{Funding.}

Acknowledgments. We wish to acknowledge technical support from X. Morvan and J.-R. Thébault, and financial support from the CNRS within the framework of a défi émergent program.

Disclosures. The authors declare no conflicts of interest.

Data availability. Data underlying the results presented in this paper are not publicly available at this time but may be obtained from the authors upon reasonable request.

\section{REFERENCES}

1. A. Eden, The Search for Christian Doppler (Springer, 1992).

2. M. Mayor, C. Lovis, and N. C. Santos, Nature 513, 328 (2014).

3. R. Adrian and C. Yao, Exp. Fluids 5, 17 (1986).

4. T. W. Hänsch and A. L. Schawlow, Opt. Commun. 13, 68 (1975).

5. R. J. Doviak and D. S. Zrnić, Doppler radar and weather observations (Courier Corporation, 2006).

6. L. Fang, M. J. Padgett, and J. Wang, Laser Photon. Rev. 11, 1700183 (2017).

7. M. Mansuripur, "Angular momentum exchange between light and material media deduced from the doppler shift," in Optical Trapping and Optical Micromanipulation IX, , vol. 8458 (International Society for Optics and Photonics, 2012), p. 845805.

8. J. Courtial, K. Dholakia, D. Robertson, L. Allen, and M. Padgett, Phys. Rev. Lett. 80, 3217 (1998).
9. M. P. Lavery, F. C. Speirits, S. M. Barnett, and M. J. Padgett, Science 341, 537 (2013).

10. M. P. Lavery, S. M. Barnett, F. C. Speirits, and M. J. Padgett, Optica 1, 1 (2014).

11. A. Y. Okulov, J. Opt. Soc. Am. B 29, 714 (2012).

12. A. Y. Okulov, Phys. Lett. A 374, 4523 (2010).

13. P. Georgi, C. Schlickriede, G. Li, S. Zhang, and T. Zentgraf, Optica 4, 1000 (2017)

14. B. Liu, H. Chu, H. Giddens, R. Li, and Y. Hao, Sci. Rep. 9, 1 (2019).

15. O. Emile, J. Emile, and C. Brousseau, Appl. Phys. Lett. 116, 221102 (2020).

16. N. Uribe-Patarroyo, A. Fraine, D. S. Simon, O. Minaeva, and A. V. Sergienko, Phys. Rev. Lett. 110, 043601 (2013).

17. G. Labroille, B. Denolle, P. Jian, P. Genevaux, N. Treps, and J.-F. Morizur, Opt. Express 22, 15599 (2014).

18. A. E. Siegman, Lasers (University Science Books, 1986).

19. J. Courtial and M. Padgett, Opt. Commun. 159, 13 (1999).

20. O. Emile and J. Emile, Appl. Phys. B 117, 487 (2014).

21. G. Xie, H. Song, Z. Zhao, G. Milione, Y. Ren, C. Liu, R. Zhang, C. Bao, L. Li, Z. Wang, K. Pang, D. Starobudov, B. Lynn, M. Tur, and A. E. Willner, Opt. Lett. 42, 4482 (2017).

22. A. Y. Okulov, JETP Lett. 88, 487 (2008).

23. I. Basistiy, V. Slyusar, M. Soskin, M. Vasnetsov, and A. Y. Bekshaev, Opt. Lett. 28, 1185 (2003).

24. A. Y. Okulov, Phys. Rev. A 80, 013837 (2009).

25. M. Padgett, Proc. Math. Phys. Eng. Sci. 470, 20140633 (2014).

26. O. Emile and J. Emile, Ann. Phys. 530, 1800111 (2018).

27. B. Thidé, H. Then, J. Sjöholm, K. Palmer, J. Bergman, T. Carozzi, Y. N. Istomin, N. Ibragimov, and R. Khamitova, Phys. Rev. Lett. 99, 087701 (2007).

28. F. Seiboth, M. Kahnt, M. Lyubomirskiy, M. Seyrich, F. Wittwer, T. Ullsperger, S. Nolte, D. Batey, C. Rau, and C. G. Schroer, Opt. Lett. 44, 4622 (2019).

29. J. Verbeeck, H. Tian, and P. Schattschneider, Nature 467, 301 (2010).

30. C. W. Clark, R. Barankov, M. G. Huber, M. Arif, D. G. Cory, and D. A. Pushin, Nature 525, 504 (2015).

31. T. Wang, M. Ke, W. Li, Q. Yang, C. Qiu, and Z. Liu, Appl. Phys. Lett. 109, 123506 (2016).

32. F. Wu, B. G. Van Schie, J. E. Keymer, and C. Dekker, Nat. Nanotech. 10, 719 (2015).

33. S. D. Karlen, H. Reyes, R. Taylor, S. I. Khan, M. F. Hawthorne, and M. A. Garcia-Garibay, Proc. Nat. Acad. Sci. 107, 14973 (2010). 


\section{FULL REFERENCES}

1. A. Eden, The Search for Christian Doppler (Springer, 1992).

2. M. Mayor, C. Lovis, and N. C. Santos, "Doppler spectroscopy as a path to the detection of earth-like planets," Nature 513, 328-335 (2014).

3. R. Adrian and C. Yao, "Power spectra of fluid velocities measured by laser doppler velocimetry," Exp. Fluids 5, 17-28 (1986).

4. T. W. Hänsch and A. L. Schawlow, "Cooling of gases by laser radiation," Opt. Commun. 13, 68-69 (1975).

5. R. J. Doviak and D. S. Zrnić, Doppler radar and weather observations (Courier Corporation, 2006).

6. L. Fang, M. J. Padgett, and J. Wang, "Sharing a common origin between the rotational and linear doppler effects," Laser Photon. Rev. 11, 1700183 (2017).

7. M. Mansuripur, "Angular momentum exchange between light and material media deduced from the doppler shift," in Optical Trapping and Optical Micromanipulation IX, vol. 8458 (International Society for Optics and Photonics, 2012), p. 845805.

8. J. Courtial, K. Dholakia, D. Robertson, L. Allen, and M. Padgett, "Measurement of the rotational frequency shift imparted to a rotating light beam possessing orbital angular momentum," Phys. Rev. Lett. 80, 3217 (1998).

9. M. P. Lavery, F. C. Speirits, S. M. Barnett, and M. J. Padgett, "Detection of a spinning object using light's orbital angular momentum," Science 341, 537-540 (2013).

10. M. P. Lavery, S. M. Barnett, F. C. Speirits, and M. J. Padgett, "Observation of the rotational doppler shift of a white-light, orbital-angularmomentum-carrying beam backscattered from a rotating body," Optica 1, 1-4 (2014).

11. A. Y. Okulov, "Rotational doppler shift of a phase-conjugated photon," J. Opt. Soc. Am. B 29, 714-718 (2012).

12. A. Y. Okulov, "Laser singular theta-pinch," Phys. Lett. A 374, 4523-4527 (2010).

13. P. Georgi, C. Schlickriede, G. Li, S. Zhang, and T. Zentgraf, "Rotational doppler shift induced by spin-orbit coupling of light at spinning metasurfaces," Optica 4, 1000-1005 (2017).

14. B. Liu, H. Chu, H. Giddens, R. Li, and Y. Hao, "Experimental observation of linear and rotational doppler shifts from several designer surfaces," Sci. Rep. 9, 1-9 (2019).

15. O. Emile, J. Emile, and C. Brousseau, "Rotational doppler shift upon reflection from a right angle prism," Appl. Phys. Lett. 116, 221102 (2020).

16. N. Uribe-Patarroyo, A. Fraine, D. S. Simon, O. Minaeva, and A. V. Sergienko, "Object identification using correlated orbital angular momentum states," Phys. Rev. Lett. 110, 043601 (2013).

17. G. Labroille, B. Denolle, P. Jian, P. Genevaux, N. Treps, and J.-F. Morizur, "Efficient and mode selective spatial mode multiplexer based on multi-plane light conversion," Opt. Express 22, 15599-15607 (2014).

18. A. E. Siegman, Lasers (University Science Books, 1986).

19. J. Courtial and M. Padgett, "Performance of a cylindrical lens mode converter for producing laguerre-gaussian laser modes," Opt. Commun. 159, 13-18 (1999).

20. O. Emile and J. Emile, "Young's double-slit interference pattern from a twisted beam," Appl. Phys. B 117, 487-491 (2014).

21. G. Xie, H. Song, Z. Zhao, G. Milione, Y. Ren, C. Liu, R. Zhang, C. Bao, L. Li, Z. Wang, K. Pang, D. Starobudov, B. Lynn, M. Tur, and A. E. Willner, "Using a complex optical orbital-angular-momentum spectrum to measure object parameters," Opt. Lett. 42, 4482-4485 (2017).

22. A. Y. Okulov, "Optical and sound helical structures in a mandelstambrillouin mirror," JETP Lett. 88, 487-491 (2008).

23. I. Basistiy, V. Slyusar, M. Soskin, M. Vasnetsov, and A. Y. Bekshaev, "Manifestation of the rotational doppler effect by use of an off-axis optical vortex beam," Opt. Lett. 28, 1185-1187 (2003).

24. A. Y. Okulov, "Twisted speckle entities inside wave-front reversal mirrors," Phys. Rev. A 80, 013837 (2009).

25. M. Padgett, "Light's twist," Proc. Math. Phys. Eng. Sci. 470, 20140633 (2014).

26. O. Emile and J. Emile, "Energy, linear momentum, and angular momentum of light: What do we measure?" Ann. Phys. 530, 1800111
(2018).

27. B. Thidé, H. Then, J. Sjöholm, K. Palmer, J. Bergman, T. Carozzi, Y. N. Istomin, N. Ibragimov, and R. Khamitova, "Utilization of photon orbital angular momentum in the low-frequency radio domain," Phys. Rev. Lett. 99, 087701 (2007).

28. F. Seiboth, M. Kahnt, M. Lyubomirskiy, M. Seyrich, F. Wittwer, T. Ullsperger, S. Nolte, D. Batey, C. Rau, and C. G. Schroer, "Refractive hard x-ray vortex phase plates," Opt. Lett. 44, 4622-4625 (2019).

29. J. Verbeeck, H. Tian, and P. Schattschneider, "Production and application of electron vortex beams," Nature 467, 301-304 (2010).

30. C. W. Clark, R. Barankov, M. G. Huber, M. Arif, D. G. Cory, and D. A. Pushin, "Controlling neutron orbital angular momentum," Nature 525, 504-506 (2015).

31. T. Wang, M. Ke, W. Li, Q. Yang, C. Qiu, and Z. Liu, "Particle manipulation with acoustic vortex beam induced by a brass plate with spiral shape structure," Appl. Phys. Lett. 109, 123506 (2016).

32. F. Wu, B. G. Van Schie, J. E. Keymer, and C. Dekker, "Symmetry and scale orient min protein patterns in shaped bacterial sculptures," Nat. Nanotech. 10, 719-726 (2015).

33. S. D. Karlen, H. Reyes, R. Taylor, S. I. Khan, M. F. Hawthorne, and M. A. Garcia-Garibay, "Symmetry and dynamics of molecular rotors in amphidynamic molecular crystals," Proc. Nat. Acad. Sci. 107, 1497314977 (2010). 